TRANSACTIONS OF THE

AMERICAN MATHEMATICAL SOCIETY

Volume 358, Number 10, October 2006, Pages 4637-4651

S 0002-9947(06)03896-7

Article electronically published on May 9, 2006

\title{
VANISHING AND NON-VANISHING OF TRACES OF HECKE OPERATORS
}

\author{
JEREMY ROUSE
}

\begin{abstract}
Using a reformulation of the Eichler-Selberg trace formula, due to Frechette, Ono and Papanikolas, we consider the problem of the vanishing (resp. non-vanishing) of traces of Hecke operators on spaces of even weight cusp forms with trivial Nebentypus character. For example, we show that for a fixed operator and weight, the set of levels for which the trace vanishes is effectively computable. Also, for a fixed operator the set of weights for which the trace vanishes (for any level) is finite. These results motivate the "generalized Lehmer conjecture", that the trace does not vanish for even weights $2 k \geq 16$ or $2 k=12$.
\end{abstract}

\section{INTRODUCTION AND STATEMENT OF RESULTS}

Let $S_{2 k}\left(\Gamma_{0}(N)\right)$ denote the finite-dimensional $\mathbb{C}$-vector space of cusp forms of weight $2 k$ on the congruence subgroup

$$
\Gamma_{0}(N)=\left\{\left(\begin{array}{ll}
a & b \\
c & d
\end{array}\right) \in S L_{2}(\mathbb{Z}): c \equiv 0 \quad(\bmod N)\right\} .
$$

For $f \in S_{2 k}\left(\Gamma_{0}(N)\right), f(z)=\sum_{m=1}^{\infty} a_{m} q^{m}, q=e^{2 \pi i z}$, the $n$th Hecke operator $T_{n}$ acts by

$$
T_{n} f(z)=\sum_{m=1}^{\infty}\left(\sum_{\substack{d \mid \operatorname{gcd}(m, n) \\ \operatorname{gcd}(d, N)=1}} d^{2 k-1} a_{m n / d^{2}}\right) q^{m} .
$$

It is easy to see that $T_{n}$ is a linear map and Proposition 35 of [5] (pg. 160) implies that $T_{n}$ maps $S_{2 k}\left(\Gamma_{0}(N)\right)$ into $S_{2 k}\left(\Gamma_{0}(N)\right)$. Let $\operatorname{Tr}_{2 k}\left(\Gamma_{0}(N), n\right)$ denote the trace of this linear map.

The study of the numbers $\operatorname{Tr}_{2 k}\left(\Gamma_{0}(N), n\right)$ is a classical problem and is the source of many conjectures and open problems. As usual, let $\Delta(z)$ be the unique normalized cusp form of weight 12 on $\Gamma_{0}(1)$ and let

$$
\Delta(z)=\sum_{n=1}^{\infty} \tau(n) q^{n}=q-24 q^{2}+252 q^{3}+\cdots
$$

be its Fourier expansion. Since $\Delta(z)$ is the unique normalized cusp form of weight 12 on $S L_{2}(\mathbb{Z})$, it is an eigenform of $T_{n}$ for all $n$ with eigenvalue $\tau(n)$. Thus,

Received by the editors July 15, 2004 and, in revised form, November 8, 2004.

2000 Mathematics Subject Classification. Primary 11F25; Secondary 11F72.

This research was supported by the NDSEG Fellowship Program, which is sponsored by the Department of Defense and the Office of Naval Research. 
$\operatorname{Tr}_{12}\left(\Gamma_{0}(1), n\right)=\tau(n)$. Lehmer conjectured in [6] that

$$
\tau(n) \neq 0
$$

for any $n \geq 1$.

There are three natural aspects to consider when studying the vanishing and non-vanishing of the function $\operatorname{Tr}_{2 k}\left(\Gamma_{0}(N), n\right)$, which are afforded by fixing two of the variables $k, N, n$ and letting the third vary. For example if $N$ and $n$ are fixed and $k$ varies, Frechette, Ono and Papanikolas show in 2 2 that the function

$$
R\left(\Gamma_{0}(N), n, x\right)=\sum_{k=1}^{\infty} \operatorname{Tr}_{2 k}\left(\Gamma_{0}(N), n\right) x^{k-1}
$$

is a rational function of $x$ and hence as $k$ varies the traces $\operatorname{Tr}_{2 k}\left(\Gamma_{0}(N), n\right)$ are governed by a linear recurrence relation. From this, it is easy to determine $p$-adic information about $\operatorname{Tr}_{2 k}\left(\Gamma_{0}(N), n\right)$, investigate the asymptotics of $\operatorname{Tr}_{2 k}\left(\Gamma_{0}(N), n\right)$ as a function of $k$, etc. The second aspect is to consider $\operatorname{Tr}_{2 k}\left(\Gamma_{0}(N), n\right)$ for a fixed $k$ and $N$ as $n$ varies. This problem can be studied using packets of Galois representations of Deligne and Serre. Deligne and Serre interpret Hecke eigenvalues as the traces of representations of $\operatorname{Gal}(\overline{\mathbb{Q}} / \mathbb{Q})$, so $\operatorname{Tr}_{2 k}\left(\Gamma_{0}(N), n\right)$ can be interpreted as the sum of traces of representations. In this direction, Serre's Theorem 15 of [13] (pg. 374) implies that for all $\delta>0$

$$
\#\{p<x: \tau(p)=0\}=O\left(\frac{x}{(\log x)^{3 / 2-\delta}}\right),
$$

so the density of primes $p$ for which $\tau(p)=0$ is zero (it is well known that $\tau(n)=0$ only if $\tau(p)=0$ for some prime $p \mid n)$. The third aspect is to fix $k$ and $n$ and let $N$ vary. This is the problem we will consider. Throughout, $k, n$ and $N$ will denote positive integers.

Our first result is that for a fixed $n$ and $2 k$ with $n$ not a square, there is an algorithm to compute the set of levels $N$ coprime to $n$ for which $\operatorname{Tr}_{2 k}\left(\Gamma_{0}(N), n\right)=0$. More specifically, one can give necessary and sufficient conditions under which $\operatorname{Tr}_{2 k}\left(\Gamma_{0}(N), n\right)=0$, based on the number of prime factors of $N$ in various residue classes. This algorithm is described explicitly in Section 3. For example, we illustrate this algorithm and characterize the set of pairs $(N, k)$ with $N$ odd for which $\operatorname{Tr}_{2 k}\left(\Gamma_{0}(N), 2\right)=0$ (see Sections 6 and 7 ).

There are a number of results that follow from this algorithm.

Theorem 1.1. Suppose that $n \geq 1$ is not a square. Then there exists an integer $m(n)$ such that if $\operatorname{Tr}_{2}\left(\Gamma_{0}(N), n\right)=0$ with $\operatorname{gcd}(N, n)=1$, then $N$ has no more than $m(n)$ distinct prime factors.

For $n$ not a square and $2 k \in\{4,6,8,10,14\}$, it is easy to find $N$ with many distinct prime factors for which $\operatorname{Tr}_{2 k}\left(\Gamma_{0}(N), n\right)=0$.

Theorem 1.2. If $n \geq 1$ is not a square and $2 k \in\{4,6,8,10,14\}$, then the proportion of primes $p$ for which $\operatorname{Tr}_{2 k}\left(\Gamma_{0}(p), n\right)=0$ is at least $\frac{1}{2^{\lfloor\sqrt{4 n}\rfloor+1}}$.

From Theorem 1.2 it follows that if $2 k \in\{4,6,8,10,14\}$ and $n$ is not a square, then there are infinitely many $N$ for which $\operatorname{Tr}_{2 k}\left(\Gamma_{0}(N), n\right)=0$. If $2 k=2$, the same result follows for a large class of $n$, where the levels $N$ are of course still subject to Theorem 1.1 . 
Theorem 1.3. Suppose that $n \geq 1$ is not a square, $f \in\{3,4,5,7,13\}$ and $\chi(n)=$ -1 , where $\chi$ is the unique, real, non-principal Dirichlet character of conductor $f$. Then there are infinitely many $N$ coprime to $n$ such that $\operatorname{Tr}_{2}\left(\Gamma_{0}(N), n\right)=0$.

This result motivates the following conjecture.

Conjecture 1.4. Suppose that $n \geq 1$ is not a square. Then there exist infinitely many $N$ coprime to $n$ such that $\operatorname{Tr}_{2}\left(\Gamma_{0}(N), n\right)=0$.

Using results similar to Theorem 1.3, the above conjecture has been verified numerically for all $n<1500$.

Regarding non-vanishing, we have Theorem 1.1 in the case of weight 2. In general, finding $N, n$ for which $\operatorname{Tr}_{2 k}\left(\Gamma_{0}(N), n\right)=0$ when $2 k=12$ or $2 k \geq 16$ is substantially harder. In fact, implementing the algorithm described in Section 3, the author has shown that for $n$ not a square, $n \leq 22$, there are no levels $N$ coprime to $n$ with $\operatorname{Tr}_{2 k}\left(\Gamma_{0}(N), n\right)=0$ for $2 k=12$, or $16 \leq 2 k \leq 100$. This motivates the following "Generalized Lehmer Conjecture".

Conjecture 1.5. If $n \geq 1$ is not a square, $\operatorname{gcd}(N, n)=1$ and $2 k=12$ or $2 k \geq 16$, then

$$
\operatorname{Tr}_{2 k}\left(\Gamma_{0}(N), n\right) \neq 0 .
$$

In Section 7, we prove this conjecture for $n=2$. This conjecture is supported by the following theorem which indicates the paucity of weights for which the trace vanishes.

Theorem 1.6. If $n \geq 1$ is not a square, then the set

$$
\left\{k \geq 1 \text { : there is an } N \text { coprime to } n \text { with } \operatorname{Tr}_{2 k}\left(\Gamma_{0}(N), n\right)=0\right\}
$$

is finite. Moreover, if $m=7 \sqrt{n}$, then there are at most $(2 m)^{36 m^{3}}$ such $k$.

Theorem 1.7. If $n \geq 1$ is not a square, then

$$
\begin{aligned}
\#\left\{0<N<x: \operatorname{gcd}(N, n)=1, \operatorname{Tr}_{2 k}\left(\Gamma_{0}(N), n\right)=0\right. \text { for some } & k 1\} \\
& =O_{n}\left(\frac{x}{\sqrt{\log x}}\right) .
\end{aligned}
$$

\section{The Eichler-Selberg trace formula}

In this section, we will state in full the reformulation of the Eichler-Selberg trace formula (specializing to our purposes) as given in 2. We will make some important observations about how the trace varies as a function of the level.

First, we will define the various rational constants appearing in the EichlerSelberg trace formula (following [4]).

Definition. Suppose that $n \geq 1$ is fixed and $s$ is an integer such that $s^{2}-4 n<0$ or $s^{2}-4 n$ is a positive square. Let $t_{0}$ be the largest positive integer such that $t_{0}^{2} \mid\left(s^{2}-4 n\right)$. Then, let

$$
t(s, n)= \begin{cases}t_{0} & \text { if }\left(s^{2}-4 n\right) / t_{0}^{2} \equiv 1 \quad(\bmod 4), \\ t_{0} / 2 & \text { if }\left(s^{2}-4 n\right) / t_{0}^{2} \equiv 2,3 \quad(\bmod 4) .\end{cases}
$$


Definition. For $n \geq 1$ fixed, $s$ an integer such that $s^{2}-4 n$ is a positive square or is negative and some $f \mid t(s, n)$, define

$$
b(s, f, n)= \begin{cases}\frac{1}{2} \phi\left(\left(s^{2}-4 n\right)^{1 / 2} / f\right) & \text { if } s^{2}-4 n>0, \\ h\left(\left(s^{2}-4 n\right) / f^{2}\right) / \omega\left(\left(s^{2}-4 n\right) / f^{2}\right) & \text { if } s^{2}-4 n<0,\end{cases}
$$

where $\phi$ denotes Euler's $\phi$-function, $h(-d)$ denotes the class number of the imaginary quadratic order $R$ of discriminant $-d$, and $\omega(-d)$ denotes half the cardinality of the group of units of $R$.

Definition. Suppose $n \geq 1$ is fixed, $\ell$ is a prime, $s$ is an integer such that $s^{2}-4 n$ is a positive square or is negative, $f \mid t(s, n)$, and $N$ is a positive integer coprime to $n$. Let $v=\operatorname{ord}_{\ell}(N)$ and let $b=\operatorname{ord}_{\ell}(f)$. Let $A$ denote the number of incongruent $\left(\bmod \ell^{v+b}\right)$ solutions to the system of congruences

$$
\begin{aligned}
\Phi(x)=x^{2}-s x+n & \equiv 0 & & \left(\bmod \ell^{v+2 b}\right), \\
2 x & \equiv s & & \left(\bmod \ell^{b}\right),
\end{aligned}
$$

and let $B$ denote the number of incongruent $\left(\bmod \ell^{v+b}\right)$ solutions to

$$
\begin{aligned}
\Phi(x)=x^{2}-s x+n & \equiv 0 & & \left(\bmod \ell^{v+2 b+1}\right), \\
2 x & \equiv s & & \left(\bmod \ell^{b}\right) .
\end{aligned}
$$

Define the integer $c(s, f, N, n, \ell)$ by

$$
c(s, f, N, n, \ell)=\left\{\begin{array}{lll}
A & \text { if }\left(s^{2}-4 n\right) / f^{2} \not \equiv 0 & (\bmod \ell), \\
A+B & \text { if }\left(s^{2}-4 n\right) / f^{2} \equiv 0 & (\bmod \ell),
\end{array}\right.
$$

and let

$$
c(s, f, N, n)=\prod_{\ell \mid N} c(s, f, N, n, \ell) .
$$

Definition. Let

$$
R\left(\Gamma_{0}(N), n ; x\right)=\sum_{k=1}^{\infty} \operatorname{Tr}_{2 k}\left(\Gamma_{0}(N), n\right) x^{k-1}
$$

denote the generating function for the trace as the weight varies.

The following is Theorem 3.3 of 2 and is the reformulation of the Eichler-Selberg trace formula we will use.

Theorem 2.1. If $N$ and $n$ are positive coprime integers and $n$ is not a square, then

$$
\begin{aligned}
R\left(\Gamma_{0}(N), n ; x\right) & =\sigma_{1}(n)+\sum_{\substack{d \mid n \\
d<\sqrt{n}}} \frac{2 d^{2}}{n-d^{2}} \sum_{f \mid\left(\frac{n}{d}-d\right)} \frac{b\left(\frac{n}{d}+d, f, n\right) c\left(\frac{n}{d}+d, f, N, n\right)}{d^{2} x-1} \\
& -\frac{1}{2} \sum_{\substack{s \in \mathbb{Z} \\
s^{2}-4 n<0}} \sum_{f \mid t(s, n)} b(s, f, n) c(s, f, N, n) \frac{n x+1}{n^{2} x^{2}+\left(2 n-s^{2}\right) x+1},
\end{aligned}
$$

where $\sigma_{1}(n)$ denotes as usual the sum of the divisors of $n$. 
Remark. Note that the only dependence on the level $N$ in the above formula is in the constants $c(s, f, N, n)$. Also, since $c(n / d+d, f, N, n)$ counts solutions to

$$
x^{2}-\left(\frac{n}{d}+d\right) x+n=(x-n / d)(x-d) \equiv 0
$$

modulo powers of primes dividing $N, c(n / d+d, f, N, n)>0$.

\section{Algorithm Determining trace Zero LeVels}

As mentioned above, the only dependence on the level $N$ in the trace formula is the constants $c(s, f, N, n)$.

Lemma 3.1. Let $c(s, f, N, n)$ denote the constants appearing in the Eichler-Selberg trace formula. If $\ell$ is a prime and $\operatorname{ord}_{\ell}\left(s^{2}-4 n\right)=e$, then for any $j \geq 1$ we have

$$
c\left(s, f, \ell^{e+1}, n\right)=c\left(s, f, \ell^{e+j}, n\right) .
$$

To describe the algorithm to determine the set of $N$ for which $\operatorname{Tr}_{2 k}\left(\Gamma_{0}(N), n\right)=$ 0 , we need some definitions.

Definition. For $n \geq 1$ not a square and a positive integer $N$ coprime to $n$, let $V(n, N)$ be the row vector whose entries are $c(s, f, N, n)$ for all $s^{2}<4 n$ and $f \mid t(s, n)$ together with $c(n / d+d, f, N, n)$ for all $d \mid n, d<\sqrt{n}$ and $f \mid(n / d-d)$.

For example,

$$
\begin{aligned}
V(3,11) & =[c(0,1,11,3), c(0,2,11,3), c(1,1,11,3), c(2,1,11,3), c(3,1,11,3), \\
& c(4,1,11,3), c(4,2,11,3)] \\
& =[0,0,1,2,0,2,2] .
\end{aligned}
$$

Definition. Say that $V\left(n, N_{1}\right)$ and $V\left(n, N_{2}\right)$ are "projectively equivalent" if there exists $\lambda \in \mathbb{Q}^{\times}$such that $V\left(n, N_{1}\right)=\lambda V\left(n, N_{2}\right)$.

Remark. Suppose $n \geq 1$ is not a square, $N_{1}$ and $N_{2}$ are coprime to $n$ and $2 k \geq 4$. If $V\left(n, N_{1}\right)$ and $V\left(n, N_{2}\right)$ are projectively equivalent, then the trace formula implies that

$$
\operatorname{Tr}_{2 k}\left(\Gamma_{0}\left(N_{1}\right), n\right)=\lambda \operatorname{Tr}_{2 k}\left(\Gamma_{0}\left(N_{2}\right), n\right) .
$$

Hence, $\operatorname{Tr}_{2 k}\left(\Gamma_{0}\left(N_{1}\right), n\right)=0$ if and only if $\operatorname{Tr}_{2 k}\left(\Gamma_{0}\left(N_{2}\right), n\right)=0$.

Definition. For $n \geq 1$ not a square and $\ell$ a prime, let

$$
M(n, \ell)=\max \left\{\max _{s^{2}<4 n}\left(\operatorname{ord}_{\ell}\left(s^{2}-4 n\right)\right), \max _{d \mid n, d<\sqrt{n}}\left(\operatorname{ord}_{\ell}(n / d-d)\right)\right\}+1
$$

if $\operatorname{gcd}(\ell, n)=1$ and there exists an $s$ such that $\ell \mid\left(s^{2}-4 n\right)$ or a $d<\sqrt{n}$ such that $\ell \mid(n / d-d)$. Otherwise, let $M(n, \ell)=0$. Let $M(n)=\prod_{\ell} \ell^{M(n, \ell)}$.

The following result is the key to the algorithm determining the set of levels $N$ with $\operatorname{gcd}(n, N)=1$ for which $\operatorname{Tr}_{2 k}\left(\Gamma_{0}(N), n\right)=0$.

Lemma 3.2. For a fixed positive integer $n \geq 1$ not a square, and any positive integer $N$ coprime to $n$, there exists $N_{0} \mid M(n)$ and $\epsilon_{s} \in\{0,1\}$ such that $V(n, N)$ is projectively equivalent to a vector whose entries are $\epsilon_{s} c\left(s, f, N_{0}, n\right)$ followed by $c\left(n / d+d, f, N_{0}, n\right)$. 
Definition. Define the sequence $d(s, 2 k, n)$ as follows:

$$
\sum_{k=1}^{\infty} d(s, 2 k, n) x^{k-1}=\frac{n x+1}{n^{2} x^{2}+\left(2 n-s^{2}\right) x+1} .
$$

Algorithm for $2 k \geq 4$.

Step I: Determine all choices of $N_{0} \mid M(n)$ and all choices of $\epsilon_{s}$ for which

$$
\begin{aligned}
& \sum_{\substack{d \mid n \\
d<\sqrt{n}}} \frac{2 d^{2 k}}{n-d^{2}} \sum_{f \mid(n / d-d)} b(n / d+d, f, n) c\left(n / d+d, f, N_{0}, n\right) \\
& +\frac{1}{2} \sum_{\substack{s \in \mathbb{Z} \\
s^{2}-4 n<0}} \epsilon_{s} \sum_{f \mid t(s, n)} b(s, f, n) c\left(s, f, N_{0}, n\right) d(s, 2 k, n)=0 .
\end{aligned}
$$

Step II: For each of these choices of $N_{0}$ and $\epsilon_{s}$, find all levels $N$ that are multiples of $N_{0}$ with $\operatorname{gcd}(N, M(n))=N_{0}$ such that $V(n, N)$ is projectively equivalent to the vector consisting of the $\epsilon_{s} c\left(s, f, N_{0}, n\right)$, together with the $c\left(n / d+d, f, N_{0}, n\right)$. This can be done as follows. First, if for some $s, c\left(s, f, N_{0}, n\right)=0$ for all $f \mid t(s, n)$, then the choice of $\epsilon_{s}$ is irrelevant. If $\epsilon_{s}=0$ and $c\left(s, f, N_{0}, n\right) \neq 0$ for some $f$, then $c(s, f, N, n)$ must be zero and hence there must be a prime $p \mid N$ coprime to $M(n)$ for which $c(s, f, N, n, p)=0$, which happens if and only if $\left(\frac{s^{2}-4 n}{p}\right)=-1$. If $\epsilon_{s}=1$ and $c(s, f, N, n) \neq 0$ for some $f$, then $c(s, f, N, n)$ must be non-zero and hence $c(s, f, N, n, p)>0$ for all $p \mid N$; therefore $\left(\frac{s^{2}-4 n}{p}\right)=0$ or 1 for all $p \mid N$. Further, if $M(n, p)>0$, then since $\operatorname{gcd}(N, M(n))=N_{0}, \operatorname{ord}_{p}(N)=\operatorname{ord}_{p}\left(N_{0}\right)$ or $\operatorname{ord}_{p}(N)>M(n, p)$, and hence $c(s, f, N, n, p)=c\left(s, f, N_{0}, n, p\right)$, by Lemma 3.1 .

In summary, let

$$
N=N_{0} N_{1} N_{2}
$$

where $N_{0}=\operatorname{gcd}(N, M(n)), \quad N_{1}=\prod_{p \mid M(n)} p^{\max \left\{\operatorname{ord}_{p}(N)-M(n, p), 0\right\}}$ and $N_{2}=$ $N /\left(N_{0} N_{1}\right)$ (so $N_{2}$ is coprime to $M(n)$ ). Then, $V(n, N)$ is projectively equivalent to the vector consisting of the $\epsilon_{s} c\left(s, f, N_{0}, n\right)$ together with the $c(n / d+d, f, N, n)$ if and only if

(1) For all $s$ with $\epsilon_{s}=0$ and $c\left(s, f, N_{0}, n\right) \neq 0$ for some $f$, there exists $p \mid N_{2}$ with $\left(\frac{s^{2}-4 n}{p}\right)=-1$.

(2) For all $s$ with $\epsilon_{s}=1$ and $c\left(s, f, N_{0}, n\right) \neq 0$ for some $f$, for all $p \mid N_{2},\left(\frac{s^{2}-4 n}{p}\right)=$ 1.

Remark. The first step of the algorithm is a finite calculation, since there are finitely many $N_{0} \mid M(n)$ and finitely many possibilities for each $\epsilon_{s}$. For each of the valid choices of $\epsilon_{s}$ and $N_{0} \mid M(n)$, the second step specifies explicitly computable finite unions of arithmetic progressions $A$ and $B$ such that $\operatorname{Tr}_{2 k}\left(\Gamma_{0}(N), n\right)=0$ (with $N$ subject to the choice of the $\epsilon_{s}$ and $N_{0}$ ) if and only if at least one of the prime factors of $N_{2}$ is in $A$ and all of the prime factors of $N_{2}$ are in $B$.

We will prove Lemma 3.2 and Lemma 3.1 and then discuss the algorithm in the weight 2 case.

The general idea in the proof of Lemma 3.2 is as follows. If $N_{0} \mid M(n)$ and $N=$ $N_{0} p^{r}$ for some $p$ coprime to $M(n)$, then $c(s, f, N, n)$ is either 0 or $2 c\left(s, f, N_{0}, n\right)$. On the other hand, if $N_{0} \mid M(n)$ and $p \mid M(n)$ are such that $\operatorname{ord}_{p}\left(N_{0}\right)=\operatorname{ord}_{p}(M(n))$, then Lemma 3.1 implies that $c\left(s, f, N_{0} p^{r}, n, p\right)=c\left(s, f, N_{0}, n, p\right)$. 
Proof of Lemma 3.2. Suppose that $N \geq 1$ is coprime to $n$. Note that if $p \mid N$ and $\operatorname{gcd}(p, M(n))=1$, then Hensel's Lemma implies that $c(s, f, N, n, p)=1+\left(\frac{s^{2}-4 n}{p}\right)$. Let $\epsilon_{s}=0$ if there is a prime $p \mid N$ coprime to $M(n)$ with $\left(\frac{s^{2}-4 n}{p}\right)=-1$, and let $\epsilon_{s}=1$ otherwise. Hence, if $\operatorname{ord}_{p}(N)=r$, then $2 \epsilon_{s} c\left(s, f, N / p^{r}, n\right)=\epsilon_{s} c(s, f, N, n)$ (and $\left.2 c\left(n / d+d, f, N / p^{r}, n\right)=c(n / d+d, f, N, n)\right)$. Thus, $V(n, N)$ is projectively equivalent to the vector whose entries are $\epsilon_{s} c\left(s, f, N / p^{r}, n\right)$ (followed by $\left.c\left(n / d+d, f, N / p^{r}, n\right)\right)$.

Hence, we may assume that if $p \mid N$, then $p \mid M(n)$. Now, if $N$ does not divide $M(n)$, then there exists $p \mid N$ with $\operatorname{ord}_{p}(N)>\operatorname{ord}_{p}(M(n))$. Let $r=\operatorname{ord}_{p}(N)-$ $\operatorname{ord}_{p}(M(n))$. Then, Lemma 3.1 implies that $c(s, f, N, n, p)=c\left(s, f, N / p^{r}, n, p\right)$. Similarly, $c(n / d+d, f, N, n, p)=c\left(n / d+d, f, N / p^{r}, n, p\right)$, and hence we may assume that $N \mid M(n)$, as desired.

Before we prove Lemma 3.1 we will recall one version of Hensel's Lemma.

Lemma 3.3 (Hensel). Suppose that $f \in \mathbb{Z}[x], x, n, k \in \mathbb{Z}$, with $0 \leq 2 k<n$. Suppose that $f(x) \equiv 0\left(\bmod p^{n}\right)$ and that $\operatorname{ord}_{p}\left(f^{\prime}(x)\right)=k$. Then if $r \geq 0$, then there exists $y \in \mathbb{Z}$ with

$$
f(y) \equiv 0 \quad\left(\bmod p^{n+r}\right), \quad x \equiv y \quad\left(\bmod p^{n-k}\right) .
$$

Moreover, $y$ is unique $\bmod p^{n-k+r}$.

Proof. This is well known. For example, it is a repeated application of the Lemma in Section 2.2 of Chapter 2 of [11] (pg. 14).

Proof of Lemma 3.1. If $x$ is a solution to $\Phi(x) \equiv 0\left(\bmod \ell^{e+1+2 b}\right)$ we will show that $x$ is unique mod $\ell^{e / 2+1+2 b}$ and that for all $r \geq 0$, there is a $y$ such that $\Phi(y) \equiv 0$ $\left(\bmod \ell^{e+1+2 b+r}\right)$ and $x \equiv y\left(\bmod \ell^{e / 2+1+2 b}\right)$. From this the result follows.

Let $b=\operatorname{ord}_{\ell}(f)$. Since $f \mid t(s, n)$ and $t(s, n)^{2} \mid\left(s^{2}-4 n\right)$, it follows that $b \leq \operatorname{ord}_{\ell}(t) \leq$ $\frac{1}{2} \operatorname{ord}_{\ell}\left(s^{2}-4 n\right)=e / 2$.

Suppose that $x \in \mathbb{Z}$ and $\Phi(x) \equiv 0\left(\bmod \ell^{e+1+2 b}\right)$. Then, we have that

$$
4 \Phi(x)=(2 x-s)^{2}-\left(s^{2}-4 n\right) \equiv 0 \quad\left(\bmod \ell^{e+1+2 b}\right) .
$$

Since $\operatorname{ord}_{\ell}\left(s^{2}-4 n\right)=e$, it follows that $\operatorname{ord}_{\ell}(2 x-s)=\operatorname{ord}_{\ell}\left(\Phi^{\prime}(x)\right)=e / 2$. Setting $n=e+1+2 b, k=e / 2$ we have that $2 k<n$, and Hensel's lemma applies. Setting $r=0$ gives that $x$ is unique $\bmod \ell^{e / 2+1+2 b}$.

From this we get that there is a $y$ with $\Phi(y) \equiv 0\left(\bmod \ell^{e+1+2 b+r}\right)$ and $y \equiv x$ $\left(\bmod \ell^{e / 2+1+2 b}\right)$ giving $2 y \equiv s\left(\bmod \ell^{2 b}\right)$. Thus, for all $r \geq 0$ there is one $y \bmod$ $\ell^{e / 2+1+2 b}$ such that $\Phi(y) \equiv 0\left(\bmod \ell^{e+1+2 b+r}\right)$. Since $e+1+b=e / 2+1+$ $2 b+(e / 2-b) \geq e / 2+1+2 b$, it follows that $y$ is unique modulo $\ell^{e+1+b}$. Thus, $c\left(s, f, \ell^{e+j}, n\right)=c\left(s, f, \ell^{e+1}, n\right)$.

Now, we will discuss the algorithm in the weight 2 case. First, we will prove Theorem 1.1 .

Proof of Theorem 1.1. Let $M$ be the number of distinct prime factors of $M(n)$. Let

$$
m=M+\operatorname{ord}_{2}\left(\sigma_{1}(n)\right)+\max \left\{3, \max _{d}\left(\operatorname{ord}_{2}\left(n-d^{2}\right)\right)\right\} .
$$

If $\ell$ is not one of the $M$ prime divisors of $M(n)$, then $c(s, f, N, n, \ell)=0$ or 2 from Hensel's Lemma and $c(n / d+d, f, N, n, \ell)=2$. In particular, $c(s, f, N, n, \ell)$ is even. 
Suppose that $\operatorname{Tr}_{2}\left(\Gamma_{0}(N), n\right)=0$ and $N$ has more than $m$ distinct prime factors. Then, the argument above shows that each $c(s, f, N, n)$ and each $c(n / d+d, f, N, n)$ is a multiple of $2^{m-M}$. Then, the trace formula has the form

$$
\begin{aligned}
\sigma_{1}(n) & =\sum_{d \mid n, d<\sqrt{n}} \sum_{f \mid(n / d-d)} \frac{2 d^{2}}{n-d^{2}} b(n / d+d, f, n) c(n / d+d, f, N, n) \\
& +\sum_{s^{2}<4 n} \sum_{f \mid t(s)} \frac{1}{2} b(s, f, n) c(s, f, N, n) .
\end{aligned}
$$

The power of two dividing the left-hand side is $\operatorname{ord}_{2}\left(\sigma_{1}(n)\right)$. Each term in the first sum on the right-hand side is a multiple of $2^{r}$, where

$$
\begin{aligned}
r & =1-\operatorname{ord}_{2}\left(n-d^{2}\right)+(m-M) \\
& \geq-\operatorname{ord}_{2}\left(n-d^{2}\right)+\operatorname{ord}_{2}\left(\sigma_{1}(n)\right)+\operatorname{ord}_{2}\left(n-d^{2}\right)+1 \\
& \geq \operatorname{ord}_{2}\left(\sigma_{1}(n)\right)+1 .
\end{aligned}
$$

Each term in the second sum is a multiple of $2^{r}$ where $r=-2+(m-M)$, since $\operatorname{ord}_{2}(b(s, f, n)) \geq-1$. Now, $r \geq-2+3+\operatorname{ord}_{2}\left(\sigma_{1}(n)\right)=\operatorname{ord}_{2}\left(\sigma_{1}(n)\right)+1$. Thus, the right-hand side is a multiple of a higher power of 2 than the left-hand side, a contradiction.

Algorithm for $2 k=2$.

Step I: Find all choices of $0 \leq a \leq m(n)$, all choices of the $\epsilon_{s}$, and all choices of $N_{0} \mid M(n)$ so that if $c(s, f, N, n)=2^{a} \epsilon_{s} c\left(s, f, N_{0}, n\right)$, then the trace is zero.

Step II: For each such solution find all $N$ coprime to $n$ with $N_{0} \mid N, \operatorname{gcd}(N, M(n))$ $=N_{0}$ for which $c(s, f, N, n)=2^{a} \epsilon_{s} c\left(s, f, N_{0}, n\right)$. Write $N=N_{0} N_{1} N_{2}$ as above. Then, $c(s, f, N, n)=2^{a} \epsilon_{s} c\left(s, f, N_{0}, n\right)$ if and only if

(1) $N_{2}$ has $a$ distinct prime factors.

(2) If $\epsilon_{s}=0$ and $c\left(s, f, N_{0}, n\right) \neq 0$ for some $f$, there exists $p \mid N_{2}$ such that $\left(\frac{s^{2}-4 n}{p}\right)=-1$.

(3) If $\epsilon_{s}=1$ and $c\left(s, f, N_{0}, n\right) \neq 0$ for some $f$, then for all $p \mid N_{2},\left(\frac{s^{2}-4 n}{p}\right)=1$.

Remark. The first step is a finite calculation, since there are finitely many choices for $N_{0}, a$ and for all the $\epsilon_{s}$. For each of the valid choices of $N_{0}, a$, and the $\epsilon_{s}$, the second step specifies explicitly computable finite unions of arithmetic progressions $A$ and $B$ such that $\operatorname{Tr}_{2}\left(\Gamma_{0}(N), n\right)=0$ (for $N$ subject to the choices of $N_{0}, a$, and the $\epsilon_{s}$ ) if and only if $N_{2}$ has a prime factors, at least one of which is in $A$ and all of which are in $B$.

This concludes the discussion of the algorithm determining the trace zero levels. See Sections 6 and 7 for the algorithm applied when $n=2$.

\section{Proofs of VANishing ReSUlts}

In this section, we will prove the results about the vanishing of $\operatorname{Tr}_{2 k}\left(\Gamma_{0}(N), n\right)$. We start with Theorem 1.2

Proof of Theorem 1.2, Let $\mathcal{S}=\left\{p\right.$ prime $: p>4 n,\left(\frac{s^{2}-4 n}{p}\right)=1$ for all $\left.s<\sqrt{4 n}\right\}$. If $p \in \mathcal{S}$, then $c(s, f, p, n)=2$ and $c(n / d+d, f, p, n)=2$ as well. Since $c(s, f, 1, n)=1$ and $c(n / d+d, f, 1, n)=1$, it follows that $V(n, p)$ is projectively equivalent to 
$V(n, 1)$. Since $\operatorname{dim} S_{2 k}\left(\Gamma_{0}(1)\right)=0$ for $2 k \in\{4,6,8,10,14\}, \operatorname{Tr}_{2 k}\left(\Gamma_{0}(1), n\right)=0$ for these $k$. The projective equivalence then implies that

$$
\operatorname{Tr}_{2 k}\left(\Gamma_{0}(p), n\right)=0 \text {. }
$$

Now, it is straightforward to verify that Dirichlet's theorem on primes in arithmetic progressions implies that if $t_{1}, \ldots, t_{a}$ are linearly independent elements of the $\mathbb{F}_{2^{-}}$ vector space $\mathbb{Q}^{\times} /\left(\mathbb{Q}^{\times}\right)^{2}$, then the density of primes $q$ for which $\left(\frac{t_{i}}{q}\right)=1$ for all $i$ is $1 / 2^{a}$. The $\mathbb{Q}^{\times} /\left(\mathbb{Q}^{\times}\right)^{2}$ subspace spanned by $s^{2}-4 n$ for $0 \leq s<\sqrt{4 n}$ has dimension at most $\lfloor\sqrt{4 n}\rfloor+1$, and from this the result follows.

Now, we will prove Theorem 1.3 This result follows from the following two more general facts.

Lemma 4.1. Suppose that $N$ and $n$ are coprime positive integers, $n$ is not a square, $\ell$ is an odd prime that divides $N,\left(\frac{n}{\ell}\right)=-1$ and $\operatorname{Tr}_{2}\left(\Gamma_{0}(N), n\right)=0$. Then,

$$
\operatorname{Tr}_{2}\left(\Gamma_{0}(\ell N), n\right)=0 .
$$

Proof. It suffices to show that

$$
c(s, f, \ell N, n, \ell)=c(s, f, N, n, \ell)
$$

and

$$
c(n / d+d, f, \ell N, n, \ell)=c(n / d+d, f, N, n, \ell),
$$

since in this case $\operatorname{Tr}_{2}\left(\Gamma_{0}(\ell N), n\right)=\operatorname{Tr}_{2}\left(\Gamma_{0}(N), n\right)=0$. Note that if $s^{2}-4 n \equiv 0$ $(\bmod \ell)$, then $s^{2} \equiv 4 n(\bmod \ell)$ and hence $\left(\frac{n}{\ell}\right)=0$ or 1 , a contradiction. Similarly, if $n / d-d \equiv 0(\bmod \ell), n \equiv d^{2}(\bmod \ell)$ and hence $\left(\frac{n}{\ell}\right)=0$ or 1 , a contradiction. The result now follows from Lemma 3.1

Lemma 4.2. Suppose that $N$ and $n$ are coprime positive integers, $n \equiv 3(\bmod 4)$, $N$ is a multiple of 16 and $\operatorname{Tr}_{2}\left(\Gamma_{0}(N), n\right)=0$. Then $\operatorname{Tr}_{2}\left(\Gamma_{0}(2 N), n\right)=0$.

Proof. It suffices to show that

$$
c(s, f, 2 N, n, 2)=c(s, f, N, n, 2)
$$

and

$$
c(n / d+d, f, 2 N, n, 2)=c(n / d+d, f, N, n, 2) .
$$

From Lemma 3.1 it suffices to show that $\operatorname{ord}_{2}(n / d-d) \leq 3$ and $\operatorname{ord}_{2}\left(s^{2}-4 n\right) \leq 3$ since the power of 2 dividing $N$ is at least 4 . If $n / d-d \equiv 0(\bmod 4)$, then $n \equiv d^{2}$ $(\bmod 4)$, a contradiction since $n \equiv 3(\bmod 4)$. Thus, $\operatorname{ord}_{2}(n / d-d) \leq 1$. If $s$ is odd, $s^{2}-4 n$ is odd, so $\operatorname{ord}_{2}\left(s^{2}-4 n\right)=0$. If $s=2 v$ is even, then $s^{2}-4 n=4 v^{2}-4 n=$ $4\left(v^{2}-n\right)$. Since $n \equiv 3(\bmod 4)$, either $v$ is even in which case $v^{2}-n$ is odd and hence $\operatorname{ord}_{2}\left(s^{2}-4 n\right)=2$, or $v$ is odd. If $v$ is odd, $v^{2} \equiv 1(\bmod 4)$, so $v^{2}-n \equiv 2$ $(\bmod 4)$. In this case $\operatorname{ord}_{2}\left(s^{2}-4 n\right)=3$. Thus, the desired result follows.

Proof of Theorem 1.3. Now, $S_{2}\left(\Gamma_{0}(N)\right)$ has dimension zero for $N \in \mathcal{S}=\{3,5,7$, $13,16\}$, and hence $\operatorname{Tr}_{2}\left(\Gamma_{0}(N), n\right)=0$ for all $N \in \mathcal{S}$ and $n$ coprime to $N$. Suppose that $q \in\{3,5,7,13\}$ and $\left(\frac{n}{q}\right)=-1$. Then $\operatorname{Tr}_{2}\left(\Gamma_{0}(q), n\right)=0$ and Lemma 4.1 imply that $\operatorname{Tr}_{2}\left(\Gamma_{0}\left(q^{m}\right), n\right)=0$ for all $m \geq 2$. If $n \equiv 3(\bmod 4)$, then $\operatorname{Tr}_{2}\left(\Gamma_{0}(16), n\right)=0$ and Lemma 4.2 imply that $\operatorname{Tr}_{2}\left(\Gamma_{0}\left(2^{m}\right), n\right)=0$ for all $m \geq 5$. 


\section{Proofs of NON-VANISHing RESUlts}

In this section we will prove Theorem 1.6 and Theorem 1.7 about the nonvanishing of $\operatorname{Tr}_{2 k}\left(\Gamma_{0}(N), n\right)$. Before we prove Theorem 1.6, we need the following lemma.

Lemma 5.1. Suppose that $R(x) \in \mathbb{Q}(x)$ is a rational function with a simple pole at $x=1$, no other poles on the unit circle, and is holomorphic at the origin. If

$$
R(x)=\sum_{r=0}^{\infty} a_{r} x^{r}
$$

is the Taylor series of $R(x)$ at $x=0$, then the set $\left\{r \geq 0: a_{r}=0\right\}$ is finite.

Proof. The coefficients $a_{r}$ are a linear recurrence sequence, since $R(x)$ is a rational function. The well-known Skolem-Mahler-Lech theorem (see 1]) states that the set of zeroes of a linear recurrence sequence is a finite union of arithmetic progressions together with a finite set. Hence, it suffices to show that there do not exist $q$ and $d$ such that $a_{q r+d}=0$ for all $r \geq 0$. Suppose to the contrary that such $q$ and $d$ exist. Then,

$$
\sum_{m \equiv d} a_{m} x^{m}
$$

is a polynomial and is therefore entire. It is easy to verify that

$$
\frac{1}{q} \sum_{b=0}^{q-1} e^{-2 \pi i d b / q} R\left(e^{2 \pi i b / q} x\right)=\sum_{m \equiv d} a_{m} x^{m} .
$$

However, since $R(x)$ has a pole at $x=1$ and no other poles on the unit circle, $R\left(e^{2 \pi i b / q} x\right)$ has a pole at $x=e^{-2 \pi i b / q}$ with no other poles on the unit circle. Therefore

$$
\frac{1}{q} \sum_{b=0}^{q-1} e^{-2 \pi i d b / q} R\left(e^{2 \pi i b / q} x\right)
$$

has a pole at $x=1$ with residue $\frac{1}{q} \operatorname{Res}(R(x), x=1) \neq 0$. This contradicts that this latter rational function is entire.

Proof of Theorem 1.6. From Lemma 3.2, if $\operatorname{Tr}_{2 k}\left(\Gamma_{0}(N), n\right)=0$ for $2 k \geq 4, n$ not a square and $\operatorname{gcd}(N, n)=1$, then there exists $N_{0} \mid M(n)$ such that the coefficient of $x^{k-1}$ in the Taylor expansion about $x=0$ of

$$
\begin{gathered}
\sigma_{1}(n)+\sum_{\substack{d \mid n \\
d<\sqrt{n}}} \frac{2 d^{2}}{n-d^{2}} \sum_{f \mid\left(\frac{n}{d}-d\right)} \frac{b\left(\frac{n}{d}+d, f, n\right) c\left(\frac{n}{d}+d, f, N_{0}, n\right)}{d^{2} x-1} \\
-\frac{1}{2} \sum_{\substack{s \in \mathbb{Z} \\
s^{2}-4 n<0}} \epsilon_{s} \sum_{f \mid t(s, n)} b(s, f, n) c\left(s, f, N_{0}, n\right) \frac{n x+1}{n^{2} x^{2}+\left(2 n-s^{2}\right) x+1}
\end{gathered}
$$

is zero. There are finitely many such rational functions, so it suffices to show that each such rational function has only finitely many coefficients that are zero.

Note that the poles of $(n x+1) /\left(n^{2} x^{2}+\left(2 n-s^{2}\right) x+1\right)$ all have absolute value $1 / n$, and that $\frac{1}{d^{2} x-1}$ has a pole at $1 / d^{2}$. Thus, since $c\left(n / d+d, f, N_{0}, n\right)>0$ for all $f \mid(n / d-d)$, each such rational function has a pole at $x=1$ and no other poles on 
the unit circle. From Lemma 5.1, it follows that each rational function of the above form only has finitely many zero coefficients.

We say that the order of a linear recurrence relation is the degree of the denominator of the corresponding rational function. A linear recurrence sequence is called "non-degenerate" if no two roots $\alpha$ and $\beta$ of the denominator of the corresponding rational function are such that $\alpha / \beta$ is a root of unity. In general, the linear recurrence sequences we consider are degenerate. However, a theorem of Berstel and Mignotte (see Theorem 1.2 of [1]) states that if a linear recurrence sequence has order $r$, then there is an $M \leq \exp \left(2 r(3 \log r)^{1 / 2}\right)$ such that all arithmetic subprogressions of common difference $M$ are identically zero or non-degenerate. Schlickewei and Schmidt prove the deep result that the number of zeroes of a non-degenerate recurrence of order $r$ (defined over $\mathbb{Q}$ ) is at most $(2 r)^{35 r^{3}}$ (see [10], Theorem 2.1, pg. 196).

Set $m=7 \sqrt{n}$. Then, there are at most $2^{m}$ choices for the $\epsilon_{s}$. Thus, there are at most $d(M(n)) 2^{m}$ rational functions. Note that if $p>4 n$, then $M(n, p)=0$. Also, if $p<4 n$, then $\left(4 n-s^{2}\right)<4 n$ and $n / d-d<4 n$. Thus, $M(n, p) \leq 1+\log (4 n) / \log (p)=$ $\log (4 n p) / \log (p)$. Thus,

$$
d(M(n)) \leq \prod_{p<4 n} \frac{\log 4 n p}{\log p} \leq \frac{4 n \log 8 n}{\log 2} \leq 49 n=m^{2} .
$$

Thus, the total number of sequences is at most $m^{2} 2^{m} \exp \left(2 m(3 \log m)^{1 / 2}\right)$. It is easy to verify that this is less than $(2 m)^{m^{3}}$. Hence, an upper bound on the number of weights $2 k$ is $(2 m)^{36 m^{3}}$, as desired.

We will now prove Theorem 1.7 .

Proof of Theorem 1.7. From Theorem [1.6 there are only finitely many $k$ for which there exists an $N$ coprime to $n$ with $\operatorname{Tr}_{2 k}\left(\Gamma_{0}(N), n\right)=0$. Thus, it suffices to show that for each pair $(k, n)$,

$$
\#\left\{N<x: \operatorname{gcd}(N, n)=1, \operatorname{Tr}_{2 k}\left(\Gamma_{0}(N), n\right)=0\right\}=O\left(\frac{x}{\sqrt{\log x}}\right) .
$$

Case I: $2 k=2$.

In this case, Theorem 1.1 implies that if $\operatorname{Tr}_{2 k}\left(\Gamma_{0}(N), n\right)=0$, then the number of distinct prime factors of $N$ is less than or equal to $m(n)$. Now, the number of $N<x$ with at most $m(n)$ distinct prime factors is asymptotic to

$$
\frac{x(\log \log x)^{m(n)-1}}{(m(n)-1) ! \log x}
$$

(see Theorem 437 of [3] ), and hence the number of $N<x$ is certainly $O(x / \sqrt{\log x}$ ).

Case II: $2 k \geq 4$.

From Lemma 3.2 it suffices to show that the number of $N<x$ with $\operatorname{Tr}_{2 k}\left(\Gamma_{0}(N), n\right)$ $=0$ projectively equivalent to some vector of the form $\epsilon_{s} c\left(s, f, N_{0}, n\right)$ followed by $c\left(n / d+d, f, N_{0}, n\right)$ for $N_{0} \mid M(n)$ is $O(x / \sqrt{\log x})$.

If $\epsilon_{s} c\left(s, f, N_{0}, n\right)=0$ for all $(s, f)$, then the trace formula gives that

$$
\operatorname{Tr}_{2 k}\left(\Gamma_{0}(N), n\right)=-\sum_{\substack{d \mid n \\ d<\sqrt{n}}} \frac{d^{2 k}}{n-d^{2}} b(n / d+d, f, n) c(n / d+d, f, N, n)<0 .
$$


If $\epsilon_{s} c\left(s, f, N_{0}, n\right)=1$ for some $(s, f)$, then no prime factors $p$ of $N$ have $\left(\frac{s^{2}-4 n}{p}\right)=$ -1 . Let $\mathcal{P}=\left\{p:\left(\frac{s^{2}-4 n}{p}\right)=-1\right\}$ and let $E=\{m: p \mid m$ for some $p \in \mathcal{P}\}$. Then, $\mathcal{P}$ has the property that

$$
\sum_{p \in \mathcal{P}} \frac{1}{p^{s}}=\frac{1}{2} \log \left(\frac{1}{1-s}\right)+\Theta(s),
$$

where $\Theta(s)$ is holomorphic. Let $E^{\prime}(x)=\#\{m<x: m \notin E\}$. Then, Theorem 2.4(a) (pg. 231) of 12 implies that $E^{\prime}(x) \sim c x / \sqrt{\log x}$ for some $c>0$. Now $\operatorname{Tr}_{2 k}\left(\Gamma_{0}(N), n\right)=0$ for $\operatorname{gcd}(N, n)=1$ implies $N \in E^{\prime}$, so

$$
\#\left\{N<x: \operatorname{Tr}_{2 k}\left(\Gamma_{0}(N), n\right)=0\right\}=O(x / \sqrt{\log x}),
$$

as desired.

\section{EXAmple OF THE ALGORITHM}

The algorithm given in Section 3 gives an explicit description of the set of odd $N$ for which $\operatorname{Tr}_{2 k}\left(\Gamma_{0}(N), 2\right)=0$. In this case, $M(2)=49$. The following table gives the set of triples $\left(2 k, N_{0},\left(\epsilon_{0}, \epsilon_{1}, \epsilon_{2}\right)\right)$ for which the trace is zero, $4 \leq 2 k \leq 14$ :

\begin{tabular}{ccc}
$2 k$ & $N_{0}$ & $\left(\epsilon_{0}, \epsilon_{1}, \epsilon_{2}\right)$ \\
\hline 4 & 1 & $(0,1,0)$ \\
4 & 1 & $(1,0,0)$ \\
4 & 1 & $(1,1,1)$ \\
6 & 1 & $(0,1,0)$ \\
6 & 1 & $(1,1,1)$ \\
8 & 1 & $(1,1,1)$ \\
10 & 1 & $(1,1,1)$ \\
14 & 1 & $(0,1,0)$ \\
14 & 1 & $(1,1,1)$
\end{tabular}

For $2 k=2, n=2, m(n)=5$, so if $N=N_{0} N_{1} N_{2}$, the upper bound on the number of prime factors $a$ of $N_{2}$ is 5 .

For $a=0, N=1$ and $N=7$ are solutions. The following is a table of all triples $\left(a, N_{0},\left(\epsilon_{0}, \epsilon_{1}, \epsilon_{2}\right)\right)$ with $a \geq 1$ for which the trace is zero:

\begin{tabular}{ccc}
$a$ & $N_{0}$ & $\left(\epsilon_{0}, \epsilon_{1}, \epsilon_{2}\right)$ \\
\hline 1 & 1 & $(0,0,1)$ \\
1 & 1 & $(1,0,0)$
\end{tabular}

This data gives the following classification of levels for which $\operatorname{Tr}_{2 k}\left(\Gamma_{0}(N), n\right)=0$. Define the following sets $A, B, C$ and $D$. Assume that $p, q$, and $r$ are primes.

$$
\begin{gathered}
A=\left\{N: N=1, N=7, \text { or } N=p^{\alpha}, p \equiv 3,5,13,19,27,45 \quad(\bmod 56)\right\} . \\
B=\{N: \text { for all } p \mid N, p \equiv 1,9,25 \quad(\bmod 56)\} . \\
C=\{N: \text { for all } p|N, p \equiv 1,2,4 \quad(\bmod 7), q| N \text { and } r \mid N \text { for some } \\
\qquad \equiv 3 \quad(\bmod 4), r \equiv 5,7 \quad(\bmod 8)\} \\
D=\{N: \text { for all } p|N, p \equiv 1,3 \quad(\bmod 8), q| N \text { and } r \mid N \text { for some } \\
q \equiv 3 \quad(\bmod 8), r \equiv 3,5,6 \quad(\bmod 7)\}
\end{gathered}
$$

The set $A$ corresponds to the two triples $(1,1,(0,0,1))$ and $(1,1,(1,0,0))$ which are solutions for $2 k=2$. The sets $B, C$, and $D$ correspond to solutions for $\left(\epsilon_{0}, \epsilon_{1}, \epsilon_{2}\right)$ 
equal to $(1,1,1),(0,1,0)$, and $(1,0,0)$, respectively. The following is a table of the set of $(2 k, N)$ with $N$ odd and $2 k \leq 14$ for which $\operatorname{Tr}_{2 k}\left(\Gamma_{0}(N), 2\right)=0$ :

\begin{tabular}{cc}
$2 k$ & $N$ \\
\hline 2 & $N \in A$ \\
4 & $N \in B \cup C \cup D$ \\
6 & $N \in B \cup C$ \\
8 & $N \in B$ \\
10 & $N \in B$ \\
14 & $N \in B \cup C$
\end{tabular}

7. Non-VANishing of $\operatorname{TR}_{2 k}\left(\Gamma_{0}(N), 2\right)$ FOR $2 k>14$

In this section we will prove the following theorem.

Theorem 7.1. If $2 k>14$ and $N$ is odd, then

$$
\operatorname{Tr}_{2 k}\left(\Gamma_{0}(N), 2\right) \neq 0 \text {. }
$$

Proof. From Lemma 3.2 any such $N$ would have $V(n, N)$ projectively equivalent to some $N_{0}=1,7$ or 49 with $\epsilon_{s}=0$ or 1 for $0 \leq s \leq 2$. As shown in the proof of Theorem 1.7 if $2 k \geq 4$ and $\epsilon_{s}=0$ for all $s<\sqrt{4 n}$, then the trace is negative. Also, for $N_{0}=49, c\left(s, f, N_{0}, 2\right)=0$ for all pairs $(s, f)$ that are relevant.

For $N_{0}=1$, there are seven choices of the $\epsilon_{s}$ for which not all the $c\left(s, f, N_{0}, 2\right)$ are zero. For $N_{0}=7$, only $c\left(1,1, N_{0}, 2\right) \neq 0$ and hence there is essentially one choice of the $\epsilon_{s}$ for which $c\left(s, f, N_{0}, 2\right)$ are not all zero. The following are the eight associated generating functions for the traces (neglecting the constant term):

\begin{tabular}{ccc}
$N_{0}$ & $\left(\epsilon_{0}, \epsilon_{1}, \epsilon_{2}\right)$ & Rational Function \\
\hline 1 & $(1,1,1)$ & $\left(96 x^{6}+24 x^{5}\right) /\left(32 x^{6}+8 x^{5}-12 x^{4}-14 x^{3}-9 x^{2}-4 x-1\right)$ \\
1 & $(0,1,1)$ & $\left(40 x^{5}-6 x^{4}+5 x^{3}+x\right) /\left(16 x^{5}-4 x^{4}-4 x^{3}-5 x^{2}-2 x-1\right)$ \\
1 & $(1,0,1)$ & $\left(16 x^{4}-4 x^{3}+2 x^{2}+x\right) /\left(8 x^{4}-4 x^{3}-2 x^{2}-x-1\right)$ \\
1 & $(1,1,0)$ & $\left(20 x^{4}+7 x^{3}-2 x^{2}-x\right) /\left(8 x^{4}+2 x^{3}-5 x^{2}-4 x-1\right)$ \\
1 & $(1,0,0)$ & $\left(3 x^{2}\right) /\left(2 x^{2}-x-1\right)$ \\
1 & $(0,1,0)$ & $\left(8 x^{3}\right) /\left(4 x^{3}-x^{2}-2 x-1\right)$ \\
1 & $(0,0,1)$ & $\left(6 x^{3}-3 x^{2}+2 x\right) /\left(4 x^{3}-4 x^{2}+x-1\right)$ \\
7 & $\left(\epsilon_{0}, 1, \epsilon_{2}\right)$ & $\left(12 x^{3}+3 x^{2}+x\right) /\left(4 x^{3}-x^{2}-2 x-1\right)$
\end{tabular}

Numbering from the top, denote these functions by $R_{1}(x), R_{2}(x), \ldots, R_{8}(x)$ and write

$$
R_{i}(x)=\sum_{r=1}^{\infty} a_{i}(r) x^{r}
$$

It is easy to check that $a_{3}(r), a_{5}(r), a_{7}(r)$ and $a_{8}(r)$ are odd for $r \geq 2$. It is also easy to check that $a_{2}(r)$ and $a_{4}(r)$ are never multiples of 11 for $r \geq 1$. Thus, it suffices to consider $R_{1}(x)$ and $R_{6}(x)$. Note that $a_{1}(r)=0$ for $r \in\{1,2,3,4,6\}$ and $a_{6}(r)=0$ for $r \in\{1,2,6\}$.

Now,

$$
R_{1}(x)-R_{2}(x)=\frac{8 x^{3}}{8 x^{3}+4 x^{2}+2 x+1}=\sum_{r=0}^{\infty} 2^{4 r+3} x^{4 r+3}-\sum_{r=0}^{\infty} 2^{4 r+4} x^{4 r+4},
$$

and hence $a_{1}(r) \equiv a_{6}(r)\left(\bmod 2^{r}\right)$. Thus, it suffices to show that $a_{6}(r) \not \equiv 0$ $\left(\bmod 2^{r}\right)$ for $r \geq 7$. 
An explicit formula for $a_{6}(r)$ is straightforward to obtain and yields that $\left|a_{6}(r)\right| \leq$ $\frac{2 \sqrt{14}}{7} 2^{r}+1$. It is easy to verify that for $r \equiv 0(\bmod 4), a_{6}(r) \equiv 8(\bmod 16)$ and for $r \equiv 3(\bmod 4), a_{6}(r) \equiv 16(\bmod 32)$. Thus, if $a_{6}(r) \equiv 0\left(\bmod 2^{r}\right)$, then $r \equiv 1,2$ $(\bmod 4)$. However, for $r \equiv 1,2(\bmod 4), a_{6}(r) \equiv 0(\bmod 3)$. Thus, the highest power of 2 dividing $a_{6}(r)$ for $r \equiv 1,2(\bmod 4)$ is at most $\frac{2 \sqrt{14}}{21} 2^{r}+1 / 3<2^{r}$. Thus, if $a_{6}(r) \equiv 0\left(\bmod 2^{r}\right)$, then $a_{6}(r)=0$, so it suffices to show that $a_{6}(r) \neq 0$ for $r \geq 7$.

Using GP to calculate $a_{6}(r)$ for $1 \leq r \leq 32000$, one can see that if $p=29201$, $29363,29873,30671,31253$ or 31721 , then $2 p+1$ is prime, the period of $a_{6}(r)$ $(\bmod 2 p+1)$ is $p$, and $a_{6}(r) \equiv 0(\bmod 2 p+1)$ if and only if $r \equiv 1,2,6(\bmod p)$. If $P$ is the product of the above seven primes, it then follows that if $a_{6}(r)=0$, then $r$ is in one of 2187 residue classes $\bmod P=2.477 \times 10^{31}$. Other than 1,2 and 6 , the smallest of these is $2.919 \times 10^{27}$. Hence, if $a_{6}(r)=0$, then $r=1,2,6$ or $r \geq 2.919 \times 10^{27}$.

Note that

$$
R_{6}(x)=2+\frac{1}{x-1}+\frac{-1-2 x}{4 x^{2}+3 x+1} .
$$

Thus, if $b(0)=-1, b(1)=1$ and $b(r)=-3 b(r-1)-4 b(r-2)$, then

$$
\sum_{r=0}^{\infty} b(r) x^{r+1}=\frac{-1-2 x}{4 x^{2}+3 x+1}
$$

and hence for $r \geq 1, a_{6}(r)=b(r+1)-1$.

In 9], Phong shows that any second order linear recurrence is a generalized Lehmer sequence and uses linear forms in logarithms to put explicit lower bounds on such sequences. Theorem 3 (pg. 205) of [9] shows that for $r>4.091 \times 10^{21}$,

$$
|b(r)|>A\left(2^{r} / r^{B}\right)
$$

for $A=0.5345$ and $B=1.735 \times 10^{25}$. From this, it follows that $|b(r)|>1$ for $r>1.567 \times 10^{27}$. Thus, for $r \geq 7,\left|a_{6}(r)\right|>0$ and $\left|a_{1}(r)\right|>0$.

\section{Concluding Remarks about conjectures}

Theorem 1.3 implies that for a large class of $n$, there are infinitely many $N$ coprime to $n$ with the property that $\operatorname{Tr}_{2}\left(\Gamma_{0}(N), n\right)=0$. For $n$ not in this set, one can search for some $N$ coprime to $n$ with $\operatorname{Tr}_{2}\left(\Gamma_{0}(N), n\right)=0$. If $N$ has a prime factor $\ell$ for which $\operatorname{ord}_{\ell}(n) \geq M(n, \ell)$, then Lemma 3.1 implies that $\operatorname{Tr}_{2}\left(\Gamma_{0}\left(N \ell^{r}\right), n\right)=0$ for all $r \geq 0$. Using this approach, we have verified Conjecture 1.4 for $n<1500$. The following is a table of the $n<300$ to which Theorem 1.3 does not apply and an infinite collection of $N$ for which $\operatorname{Tr}_{2}\left(\Gamma_{0}(N), n\right)=0$ :

\begin{tabular}{cccccc}
$n$ & $N$ & $n$ & $N$ & $n$ & $N$ \\
\hline 30 & $73^{v}$ & 130 & $11 \cdot 31^{v}$ & 205 & $4 \cdot 19^{v}$ \\
105 & $17^{v}$ & 156 & $17^{v}$ & 246 & $19^{v}$ \\
114 & $17^{v}$ & 165 & $17^{v}$ & 261 & $11^{v}$ \\
120 & $11^{v}$ & 186 & $19^{v}$ & 270 & $41^{v}$ \\
126 & $19^{v}$ & 204 & $37 \cdot 11^{v}$ & 274 & $19^{v}$
\end{tabular}

Remark. One could prove Conjecture 1.4 for a larger class of $n$ by noting that $S_{2}\left(\Gamma_{0}(p)\right)$ is one dimensional and is hence spanned by a Hecke eigenform for $p \in$ $\{11,17,19\}$. Then, if $\operatorname{Tr}_{2}\left(\Gamma_{0}(p), \ell\right)=0$ and $\operatorname{ord}_{\ell}(n)=1$, then $\operatorname{Tr}_{2}\left(\Gamma_{0}(p), n\right)=0$. In this case, $\left(\frac{n}{p}\right)=-1$ would guarantee that $\operatorname{Tr}_{2}\left(\Gamma_{0}\left(p^{r}\right), n\right)=0$ for all $r \geq 2$. 


\section{ACKNOWLEDGEMENTS}

The author is grateful to Ken Ono for his assistance. The author used GP version 2.0.20 for computations. The author would like to thank the referee for his helpful comments.

\section{REFERENCES}

1. G. Everest, A. van der Poorten, I. Shparlinski, and T. Ward, Recurrence sequences, Mathematical Surveys and Monographs, vol. 104, American Mathematical Society, Providence, RI, 2003. MR $1990179(2004 \mathrm{c}: 11015)$

2. S. Frechette, K. Ono, and M. Papanikolas, The combinatorics of traces of Hecke operators, Proc. Natl. Acad. Sci. 101 (2004), 17016-17020. MR2114776 (2005h:11090)

3. G. H. Hardy and E. M. Wright, An introduction to the theory of numbers, The Clarendon Press, Oxford University Press, New York, 1979. MR0568909 (81i:10002)

4. H. Hijikata, A. Pizer, and T. Shemanske, The basis problem for modular forms on $\Gamma_{0}(N)$, Proc. Japan Acad. Ser. A Math. Sci. 56 (1980), no. 6, 280-284. MR0581471 (81k:10045)

5. N. Koblitz, Introduction to elliptic curves and modular forms, Graduate Texts in Mathematics, vol. 97, Springer-Verlag, New York, 1993. MR1216136 (94a:11078)

6. D. H. Lehmer, The vanishing of Ramanujan's function $\tau(n)$, Duke Math. J. 14 (1947), 429433. MR0021027 (9:12b)

7. K. Ono, The web of modularity: arithmetic of the coefficients of modular forms and q-series, CBMS Regional Conference Series in Mathematics, vol. 102, Published for the Conference Board of the Mathematical Sciences, Washington, DC, 2004. MR2020489 (2005c:11053)

8. The PARI Group, Bordeaux, Pari/gp, version 2.0.20, 1999, available from http://pari.math.u-bordeaux.fr/.

9. B. M. Phong, On generalized Lehmer sequences, Acta Math. Hungar. 57 (1991), no. 3-4, 201-211. MR1139314 (92m:11021)

10. H. P. Schlickewei and W. M. Schmidt, The number of solutions of polynomial-exponential equations, Compositio Math. 120 (2000), no. 2, 193-225. MR1739179 (2001b:11022)

11. J.-P. Serre, A course in arithmetic, Springer-Verlag, New York, 1973. MR0344216 (49:8956)

12. _ Divisibilité de certaines fonctions arithmétiques, Enseignement Math. (2) 22 (1976), no. 3-4, 227-260. MR0434996 (55:7958)

13. Q Quelques applications du théorème de densité de Chebotarev, Inst. Hautes Études Sci. Publ. Math. (1981), no. 54, 323-401. MR0644559(83k:12011)

Department of Mathematics, University of Wisconsin, Madison, Wisconsin 53706

E-mail address: rouse@math.wisc.edu 